\title{
A ESCOLA DE ENFERMAGEM DE RIBEIRÃO PRETO, DA USP, E A SOCIEDADE HONORÍFICA DE ENFERMAGEM PRÓ SIGMA THETA TAU INTERNACIONAL
}

Maria Helena Larcher Caliri ${ }^{1}$

Carla A. Arena Ventura ${ }^{2}$

Caliri MHL, Ventura CAA. A Escola de Enfermagem de Ribeirão Preto, da USP, e a Sociedade Honorífica de Enfermagem Pró Sigma Theta Tau Internacional. Rev Latino-am Enfermagem 2002 março-abril; 10(2):239-40.

A Sociedade Honorifica da FIERP - Pró Sigma Theta Tau Internacional será transformada em um capítulo da Sociedade Internacional de Enfermagem Sigma Theta Tau Internacional, a partir de 2002. Após uma série de dificuldades e desafios, o Brasil terá o primeiro capítulo da STTI. A possibilidade de participar dessa rede internacional de enfermagem será de grande importância para a enfermagem brasileira e da América Latina.

DESCRITORES: enfermagem, rede mundial, sociedades de enfermagem

\section{THE UNIVERSITY OF SÃO PAULO AT RIBEIRÃO PRETO COLLEGE OF NURSING AND THE FIERP HONOR SOCIETY "PRO SIGMA THETA TAU INTERNATIONAL"}

The FIERP Honor Society will become a Sigma Theta Tau International chapter in 2002. The Honor Society had to face significant challenges, but the opportunity to participate in this international network will be of great importance to Brazilian and Latin American Nursing.

DESCRIPTORS: nursing, global network, nursing societies

\section{LA ESCUELA DE ENFERMERÍA DE RIBEIRÃO PRETO/USP, Y LA SOCIEDAD HONORÍFICA DE ENFERMERÍA PRÓ SIGMA THETA TAU INTERNACIONAL}

La Sociedad Honorífica de la FIERP - Pro Sigma Theta Tau Internacional será transformada en un capitulo de la Sociedad Internacional de Enfermería Sigma Theta Tau Internacional (STTI) a partir de 2002. Después de una serie de dificultades y desafíos, el Brasil va a crear el primer capítulo de la STTI. La posibilidad de participar de ésta red internacional de enfermería va a ser de gran importancia para la enfermería brasileña y de América Latina.

DESCRIPTORES: enfermería, red mundial, sociedad de enfermería

\footnotetext{
${ }^{1}$ Coordenadora do Comitê de Elegibilidade da Sociedade Honorífica da FIERP - Pró Sigma Theta Tau Internacional Brasil e Professor Doutor, e-mail: mhcaliri@eerp.ups.br; ${ }^{2}$ Especialista em Extensão e Cooperação Universitária. Escola de Enfermagem de Ribeirão Preto da Universidade de São Paulo, Centro Colaborador da OMS para o desenvolvimento da pesquisa em enfermagem
} 
Desde 1996, a Escola de Enfermagem de Ribeirão Preto, da USP, (EERP-USP)/Centro Colaborador da Organização Mundial da Saúde para o Desenvolvimento da Pesquisa em Enfermagem, vem mantendo contato com a Sociedade Honoríica de Enfermagem Sigma Theta Tau Internacional (STTI), com o objetivo de estreitar os laços com a enfermagem internacional.

Assim, após uma série de consultas sobre a viabilidade da formação de uma Sociedade Honorífica no âmbito da Universidade de São Paulo, optou-se pela alteração do Regimento da Fundação Instituto de Enfermagem de Ribeirão Preto (FIERP), incluindo em seu estatuto uma função honorífica e interligando a STTI e a EERPUSP por meio da FIERP. Cabe também ressaltar a participação da Escola de Enfermagem da USP na composição dos Comitês de trabalho da Sociedade Honorífica da FIERP, a partir de sua constituição.

Em 2000, foi realizada a primeira Cerimônia de Nomeação da Sociedade Honoríica da FIERP Pró-Sigma Theta Tau Internacional Brasil, totalizando 126 membros (43 alunos de graduação, 17 alunos de pós-graduação, 05 ex-alunos e 61 líderes da comunidade). No primeiro ano da Sociedade Honoríica, foram realizadas 08 atividades cientificas destinadas aos seus membros.

Em março de 2001, a Sociedade Pró-Sigma realizou a segunda Cerimônia de Nomeação, introduzindo outros 61 membros (19 alunos de graduação, 11 alunos de pós-graduação, 07 ex-alunos e 24 líderes da comunidade). Ainda em 2001, foram oferecidas várias atividades exclusivas aos membros.

No final de março, a Sociedade foi avaliada por uma Consultora Internacional da STTI, a Enfa. Lola Fehr, que verificou a possibilidade de formação de um capítulo no Brasil. Em julho de 2001, a Sociedade da FIERP foi aprovada pelo Conselho Diretor da STTI e, em novembro, apresentou sua proposta formal durante a Convenção Bienal, realizada em Indianápolis, EUA.

Estima-se, dessa forma, que o Capítulo da STTI será instalado na FIERP, no início de 2002.

A confirmação da formação desse capítulo leva-nos a constatar que o primeiro passo de um grande desafio foi cumprido. Após 05 anos de negociações internas e externas, vislumbramos a formação do primeiro capítulo da Sociedade Honoríica Sigma Theta Tau no Brasil e na América Latina. Todavia, temos ciência de que nosso trabalho não termina aqui. Pelo contrário, agora temos o grande desafio de manter nossos membros e aumentar o intercâmbio em âmbito nacional e regional.

Nesse sentido e com o objetivo de divulgar as atividades da Sociedade Honorífica Sigma Theta Tau Internacional, apresentaremos, brevemente, o seu histórico e os benefícios oferecidos aos membros.

A Sociedade Honorifica Sigma Theta Tau Internacional foi criada em 1922, como resultado da iniciativa de 06 enfermeiras, Mary Tolle, Edith Moore, Marie Hippensteel, Dorothy Garrigus, Elizabeth Russel e Elizabeth McWilliams, todas alunas da Universidade de Indiana. Suas fundadoras escolheram as iniciais das letras gregas "Storge" - amor, "Tharos" - coragem e "Tima" - honra para compor o nome da Sociedade. Em 1936, a Sociedade Honoríica já era a primeira organização dos EUA a prover fundos para a pesquisa em Enfermagem. Nos anos 90, a Sociedade estabeleceu um plano estratégico de 10 anos, incluindo o objetivo de romper as fronteiras dos EUA e tornar-se internacional, estabelecendo uma agenda global para a organização.

Atualmente, a STTI é composta por cerca de 200.000 membros provenientes de 90 nações distintas e pertencentes a 503 sociedades honoríficas localizadas nos EUA, Austrália, Canadá, Coréia, Paquistão, Porto Rico e Taiwan. Os membros são convidados e aceitos pelos Comitês de Elegibilidade de cada capítulo e devem preencher os requisitos de excelência exigidos pela Sigma.

As atividades da Sociedade Honorifica de Enfermagem Sigma Theta Tau Internacional são dedicadas à melhoria da saúde da população através do desenvolvimento científico da prática da enfermagem. Nessa perspectiva, os membros devem estar envolvidos com a excelência da prática clínica, educação, pesquisa e liderança em enfermagem.

Para a consecução de sua missão, a STTI apóia o uso de diferentes experiências, perspectivas e conhecimentos, com o objetivo de influenciar o desenvolvimento da profissão.

Dessa forma, os membros dos Capítulos têm acesso ao Banco de Dados, com informações sobre a enfermagem e trabalhos de pesquisa, por meio da Biblioteca "Virginia Henderson" ou do Diretório de Pesquisadores de Enfermagem. Recebem, também, as revistas "Reflections" e "Image: Journal of Nursing Scholarship" editadas pela STTI, participam de uma rede com editores e assessores, para o desenvolvimento de projetos conjuntos internacionais, e são envolvidos em programas de desenvolvimento de habilidades, para a elaboração de artigos científicos.

Não podemos deixar de mencionar ainda que, em virtude da amplitude de experiências proporcionadas pela participação em uma Sociedade do porte e relevância da STTI, os membros podem participar de diversos programas oferecidos pela organização, resultando no desenvolvimento de lideranças locais e internacionais.

A formação de um capítulo da Sociedade Honorífica de Enfermagem Sigma Theta Tau Internacional no Brasil será de suma importância para a Enfermagem brasileira, pois possibilitará um maior intercâmbio com enfermeiros de outros países, por meio da participação em uma rede de excelência em enfermagem, além de favorecer a visibilidade das ações de enfermagem já realizadas no país. 